\title{
PRESENTATIONS OF NET MAPS
}

\author{
WILLIAM FLOYD, WALTER PARRY, AND KEVIN M. PILGRIM
}

\begin{abstract}
A branched covering $f: S^{2} \rightarrow S^{2}$ is a nearly Euclidean Thurston (NET) map if each critical point is simple and its postcritical set has exactly four points. We show that up to equivalence, each NET map admits a normal form in terms of simple affine data. This data can then be used as input for algorithms developed for the computation of fundamental invariants, now systematically tabulated in a large census.
\end{abstract}

\section{Contents}

1. Introduction

Acknowledgements

2. Lattices, tori, spheres, and affine maps 3

3. From presentations and diagrams to maps 4

4. Affine twisting of diagrams 6

5. Presentation and equivalence of Euclidean NET maps 7

6. Preliminary presentations of general NET maps 8

7. The effect of twisting on preliminary data

8. NET map presentations 11

9. An algorithm for computing NET map presentations 13

References

\section{INTRODUCTION}

This paper is part of our program to thoroughly investigate nearly Euclidean Thurston (NET) maps, introduced in [3]. A Thurston map $f:\left(S^{2}, P_{f}\right) \rightarrow\left(S^{2}, P_{f}\right)$ with postcritical set $P_{f}$ is NET if each critical point is simple and \# $P_{f}=4$. The set of NET maps contains the exceptional set of Euclidean NET maps as a proper subset. Euclidean NET maps are the usual exceptional set of maps whose canonical minimal associated orbifolds in the sense of [4] are Euclidean and have signature $(2,2,2,2)$. In contrast, typical NET maps have hyperbolic orbifolds.

This paper addresses the problem of succinctly describing a NET map in a computationally effective manner. Such a description is achieved by what we call a NET map presentation diagram, a graphical representation of what we call a NET map presentation. In Section 3 , we show that each of these simple diagrams $\mathbf{D}$ drawn on standard graph paper, like that shown in Figure 1, determines a NET map. Our first main result shows that every NET map arises in this way:

Date: August 6, 2018.

2010 Mathematics Subject Classification. Primary: 36F10; Secondary: 57M12.

Key words and phrases. Thurston map, branched covering. 


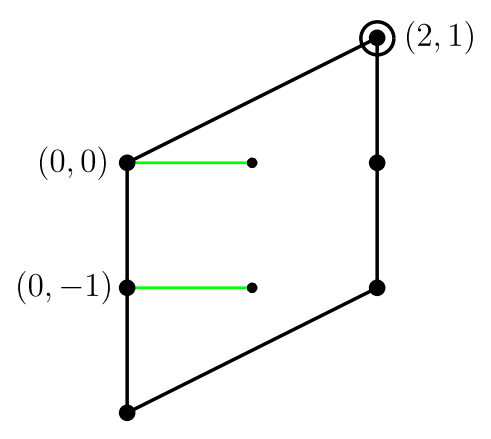

FiguRE 1. A NET map presentation diagram for Douady's rabbit quadratic polynomial

Theorem 1.1. Every NET map is Thurston equivalent to one given by a NET map presentation diagram.

Though far from unique, such presentation diagrams are nonetheless useful for two reasons.

First, they give simple input data for algorithms for the computation of fundamental invariants. Parry, with initial assistance from Floyd, wrote and continues to improve a computer program NETmap which takes as input NET map presentations and outputs a wealth of information about the NET maps. Much of our interest in NET maps lies in their computational tractability.

Second, there is a correspondence between twists of NET map presentation diagrams and twists of NET maps. The set of NET map presentation diagrams $\mathbf{D}$ admits a natural free action by the monoid $\operatorname{Mat}^{+}(2, \mathbb{Z})$ of all $2 \times 2$ matrices of integers with positive determinant; the action of $A \in \operatorname{Mat}^{+}(2, \mathbb{Z})$ is to simply transform the diagram by $A$. If we "projectivize" presentation diagrams by identifying $\mathbf{D}$ with $-\mathbf{D}$, then we obtain a free action of $\mathrm{PMat}^{+}(2, \mathbb{Z})=\operatorname{Mat}^{+}(2, \mathbb{Z}) /\{ \pm 1\}$ on projective classes $[\mathbf{D}]=\{\mathbf{D},-\mathbf{D}\}$ of presentation diagrams. In particular, we obtain a free action of $\operatorname{PSL}(2, \mathbb{Z})$ on projective classes of presentation diagrams. This action has an interpretation as functional post-composition, which we now describe. (In what follows, the subscript "2" serves to later distinguish the constructions from closely related ones.) Let $\Gamma_{2}=\left\{x \mapsto \pm x+2 \lambda: \lambda \in \mathbb{Z}^{2}\right\}$, let $S_{2}^{2}=\mathbb{R}^{2} / \Gamma_{2}$, let $\pi_{2}: \mathbb{R}^{2} \rightarrow S_{2}^{2}$ be the natural projection and let $P_{2}=\pi_{2}\left(\Lambda_{2}\right) \subset S_{2}^{2}$. The group $\operatorname{PSL}(2, \mathbb{Z})$ is naturally identified with the stabilizer of $\pi_{2}(0)$ in the mapping class group of $\left(S_{2}^{2}, P_{2}\right)$. Similarly, it follows from Section 5 that $\operatorname{PMat}^{+}(2, \mathbb{Z}) \backslash \operatorname{PSL}(2, \mathbb{Z})$ is naturally identified with the set of all Euclidean NET maps with postcritical set $P_{2}$ which fix $\pi_{2}(0)$. On the other hand, a projective class $[\mathbf{D}]$ of presentation diagrams determines a well-defined isotopy class of Thurston maps $F:\left(S_{2}^{2}, P_{2}\right) \rightarrow\left(S_{2}^{2}, P_{2}\right)$ with postcritical set $P_{2}$ (Lemma 3.3). We show:

Theorem 1.2. Suppose $A \in \operatorname{Mat}^{+}(2, \mathbb{Z})$ sends $[\mathbf{D}]$ to $\left[\mathbf{D}^{\prime}\right]$. Let $F, F^{\prime}:\left(S_{2}^{2}, P_{2}\right) \rightarrow\left(S_{2}^{2}, P_{2}\right)$ be the corresponding Thurston maps. Then $F^{\prime}=A_{2} \circ F$, where $A_{2}:\left(S_{2}^{2}, P_{2}\right) \rightarrow\left(S_{2}^{2}, P_{2}\right)$ is the map induced by $A$.

We prove Theorem 1.2 in Section 4. We also obtain the corresponding result for translations in Theorem 4.4. Together, they give a correspondence between twists of projective classes of NET map presentation diagrams and twists of NET maps by the full modular group of $F$ as well as by all Euclidean NET maps with the same postcritical set as $F$.

Outline. \$2 collects some notation and technical results. \$3 defines NET map presentation diagrams precisely and shows how a NET map presentation diagram determines a 
NET map. \$4 proves Theorem 1.2 and the supplementary Theorem 4.4, which deals with translations.

The remainder of the paper is devoted to showing that general NET maps admit NET map presentations. The basic idea is to show first that an arbitrary NET map $f$ has the form $f=h^{\prime} \circ g$ where $g$ is a Euclidean NET map as above and where now $h^{\prime}$ is a push-point homeomorphism along a set of possibly very complicated pairwise disjoint arcs. The proof of Theorem 1.1 then exploits the fact that $h^{\prime}$ can be simplified at the expense of modifying the affine map $g$.

$\$ 5$ discusses Euclidean NET maps in detail and shows that they arise as compositional factors of general NET maps. \$6 and $\$ 7$ attach preliminary data to NET maps and show how this changes under twisting. \$8 proves our main result. The proof we give of Theorem 1.1. while constructive in principle, is nevertheless somewhat implicit. In Section 9 we give an algorithm for finding NET map presentations, and illustrate it for two examples: the Douady rabbit quadratic polynomial, and the cubic rational map studied by Lodge 9].

Following this paper, [8] investigates modular groups, Hurwitz classes and dynamic portraits of NET maps. Next, [7] is partly a survey of what we know, but mostly a compilation of special NET map results, both theoretical and computational. The NET map web site [11] contains papers, a census of many examples, and executable files for the computer program NETmap that generated the census data.

Acknowledgements. The authors gratefully acknowledge support from the American Institute for Mathematics. Kevin Pilgrim was also supported by Simons grant \#245269.

\section{LATTICES, TORI, SPHERES, AND AFFINE MAPS}

A lattice $\Lambda<\mathbb{R}^{2}$ determines a group of isometries $\Gamma_{\Lambda}:=\{x \mapsto 2 \lambda \pm x: \lambda \in \Lambda\}$ generated by 180-degree rotations about elements of $\Lambda$ and a quotient map $\pi_{\Lambda}: \mathbb{R}^{2} \rightarrow \mathbb{R}^{2} / \Gamma_{\Lambda}$. Given an ordered basis $(\lambda, \mu)$ for $\Lambda$, the parallelogram in $\mathbb{R}^{2}$ spanned by $2 \lambda$ and $2 \mu$ is a fundamental domain for the quotient torus $\mathbb{R}^{2} / 2 \Lambda$; the parallelogram spanned by $2 \lambda$ and $\mu$ is a fundamental domain $F_{\Lambda}$ for the quotient sphere $\mathbb{R}^{2} / \Gamma_{\Lambda}$.

The following two lemmas will be used repeatedly in the sequel; the proofs are straightforward and are omitted.

Lemma 2.1. Let $\Lambda$ and $\Lambda^{\prime}$ be lattices in $\mathbb{R}^{2}$. Let $\Gamma$, respectively $\Gamma^{\prime}$, be the groups of Euclidean isometries of the form $x \mapsto 2 \lambda \pm x$ for some $\lambda \in \Lambda$, respectively $\lambda \in \Lambda^{\prime}$. Also let $\pi: \mathbb{R}^{2} \rightarrow$ $\mathbb{R}^{2} / \Gamma$ and $\pi^{\prime}: \mathbb{R}^{2} \rightarrow \mathbb{R}^{2} / \Gamma^{\prime}$ be the canonical quotient maps. Let $\Phi: \mathbb{R}^{2} \rightarrow \mathbb{R}^{2}$ be an affine isomorphism such that $\Phi(\Lambda) \subseteq \Lambda^{\prime}$. Then $\Phi$ induces a branched covering map $\phi: \mathbb{R}^{2} / \Gamma \rightarrow$ $\mathbb{R}^{2} / \Gamma^{\prime}$ such that $\phi \circ \pi=\pi^{\prime} \circ \Phi$ as in Figure 2. The map $\Phi$ preserves orientation if and only if $\phi$ preserves orientation. The set $\Phi(\Lambda)$ is a coset of a sublattice $\Lambda^{\prime \prime}$ of $\Lambda^{\prime}$, and the degree of $\phi$ equals the index $\left[\Lambda^{\prime}: \Lambda^{\prime \prime}\right]$.

Lemma 2.2. Let $\Lambda$ and $\Lambda^{\prime}$ be lattices in $\mathbb{R}^{2}$. Let $\Gamma$, respectively $\Gamma^{\prime}$, be the group of Euclidean isometries of the form $x \mapsto 2 \lambda \pm x$ for some $\lambda \in \Lambda$, respectively $\Lambda^{\prime}$. Also let $\pi: \mathbb{R}^{2} \rightarrow \mathbb{R}^{2} / \Gamma$ and $\pi^{\prime}: \mathbb{R}^{2} \rightarrow \mathbb{R}^{2} / \Gamma^{\prime}$ be the canonical quotient maps. Let $\phi: \mathbb{R}^{2} / \Gamma \rightarrow \mathbb{R}^{2} / \Gamma^{\prime}$ be a branched covering map such that $\phi(\pi(\Lambda)) \subseteq \pi^{\prime}\left(\Lambda^{\prime}\right)$. Then we have the following three statements.

(1) There exists a homeomorphism $\Phi: \mathbb{R}^{2} \rightarrow \mathbb{R}^{2}$ such that the restriction of $\Phi$ to $\Lambda$ is affine and $\phi \circ \pi=\pi^{\prime} \circ \Phi$. If $\pi^{\prime}(0) \in \phi(\pi(\Lambda))$, then $\Phi(\Lambda)$ is a sublattice of $\Lambda^{\prime}$. 


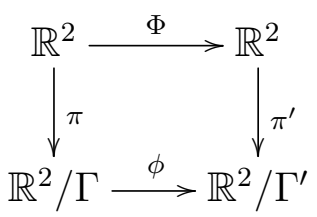

Figure 2. The maps of Lemmas 2.1 and 2.2

(2) There exists an affine isomorphism $\Psi: \mathbb{R}^{2} \rightarrow \mathbb{R}^{2}$ such that the branched map of Lemma 2.1 which $\Psi$ induces from $\mathbb{R}^{2} / \Gamma$ to $\mathbb{R}^{2} / \Gamma^{\prime}$ is $\phi$ up to isotopy rel $\pi(\Lambda)$. If $\pi^{\prime}(0) \in \phi(\pi(\Lambda))$, then $\Psi(\Lambda)$ is a sublattice of $\Lambda^{\prime}$.

(3) The maps $\Phi$ and $\Psi$ are unique up to precomposing with an element of $\Gamma$. They are also unique up to postcomposing with an element of $\Gamma^{\prime}$.

\section{From PRESENTATIONS AND DIAGRAMS TO MAPS}

In all that follows, $\Lambda_{2}$ will denote the standard integer lattice $\mathbb{Z}^{2}<\mathbb{R}^{2}$, and $\Phi: \mathbb{R}^{2} \rightarrow \mathbb{R}^{2}$ will denote an affine map $x \mapsto A x+b$ with the property that $\Lambda_{1}:=\Phi\left(\Lambda_{2}\right)$ is a proper sublattice of $\Lambda_{2}$. For $i=1,2$ we denote by $\pi_{i}$ the corresponding quotient map as in $\S 2$ and by $S_{i}^{2}$ the corresponding quotient sphere; we put $P_{i}:=\pi_{i}\left(\Lambda_{i}\right)$. The sphere $S_{i}^{2}$ has an affine structure, making it a half-translation sphere with set of corner points $P_{i}$.

Precisely described, the data of a NET map presentation with associated diagram D consists of three things. Here are the first two:

(1) An ordered basis $\left(\lambda_{1}, \lambda_{2}\right)$ for $\Lambda_{1}$. This is indicated in $\mathbf{D}$ by drawing a parallelogram $F_{1}$ with sides joining the origin to lattice points $2 \lambda_{1}, \lambda_{2}$. The choice between the two possible orderings of the basis elements is uniquely determined by the condition that the determinant of the column matrix $A=\left[\lambda_{1}, \lambda_{2}\right]$ is positive. The intersection $\left\{0, \lambda_{1}, 2 \lambda_{1}, \lambda_{2}, \lambda_{1}+\lambda_{2}, 2 \lambda_{1}+\lambda_{2}\right\}$ of $\Lambda_{1}$ with $F_{1}$ is indicated in $\mathbf{D}$ by large solid dots; note that $P_{1}=\pi_{1}\left(\Lambda_{1} \cap F_{1}\right)$.

(2) An element $b \in\left\{0, \lambda_{1}, \lambda_{2}, \lambda_{1}+\lambda_{2}\right\}$. It is indicated in $\mathbf{D}$ by circling the corresponding point in the boundary of the parallelogram $F_{1}$.

The data of (1) and (2) above determines uniquely an orientation-preserving affine isomorphism $\Phi: \mathbb{R}^{2} \rightarrow \mathbb{R}^{2}$ sending $\Lambda_{2}$ isomorphically to $\Lambda_{1}$ and sending the origin to $b$; thus it is linear iff $b=0$.

From $\S 2$, we have the following. Here, $\mathbb{R}_{1}^{2}=\mathbb{R}_{2}^{2}=\mathbb{R}^{2}$; the subscripts are for clarity. We set $S_{i}^{2}=\mathbb{R}_{i}^{2} / \Gamma_{i}$ for $i=1,2$. The identity map id $: \mathbb{R}_{1}^{2} \rightarrow \mathbb{R}_{2}^{2}$ induces a degree $d:=\left[\Lambda_{2}: \Lambda_{1}\right]$ orientation-preserving branched covering $\overline{\mathrm{id}}:\left(S_{1}^{2}, P_{1}\right) \rightarrow\left(S_{2}^{2}, P_{2}\right)$. (Not only do we have that $\overline{\mathrm{id}}\left(P_{1}\right) \subseteq P_{2}$ but even that $\overline{\mathrm{id}}\left(\pi_{1}\left(\Lambda_{2}\right)\right)=P_{2}$.) The affine isomorphism $\Phi: \mathbb{R}_{2}^{2} \rightarrow \mathbb{R}_{1}^{2}$ induces an isomorphism $\phi:\left(S_{2}^{2}, P_{2}\right) \rightarrow\left(S_{1}^{2}, P_{1}\right)$. The composition $\phi \circ \overline{\mathrm{id}}:\left(S_{1}^{2}, P_{1}\right) \rightarrow\left(S_{1}^{2}, P_{1}\right)$, is a Euclidean Thurston map, $g$. See Figure 3. The lower vertical arrows are the identity map.

We call the data in (1) and (2) above Euclidean NET map data. In the following \$5, we show that up to equivalence, every Euclidean NET map arises in this way.

The third and final ingredient in a NET map presentation is the following. Recall that the four corners of the half-translation sphere form the set $P_{1}=\pi_{1}\left(\Lambda_{1}\right)$, and that $\Lambda_{1} \cap$ $F_{1}=\left\{0, \lambda_{1}, 2 \lambda_{1}, \lambda_{2}, \lambda_{1}+\lambda_{2}, 2 \lambda_{1}+\lambda_{2}\right\}$. For convenience of exposition, we denote $P_{1}=$ $\left\{p_{1}, p_{2}, p_{3}, p_{4}\right\}$; the choice of such a labeling is not part of the data of a NET map presentation. 


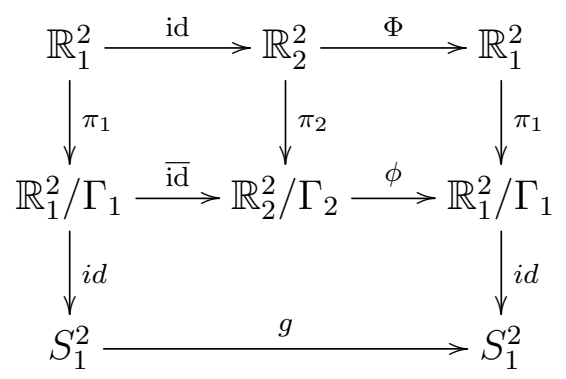

FiguRE 3. Defining $g$

(3) For each $p_{j} \in P_{1}$, a "green" (local) geodesic arc $\beta_{j}$ joining $p_{j}$ to an element of $\pi_{1}\left(\Lambda_{2}\right)$, such that the geodesics $\beta_{j}$ are pairwise disjoint. We allow the case of degenerate (zero length) geodesics. We require that every $\beta_{j}$ lifts to a line segment in $F_{1}$. The choice of the $\beta_{j}$ 's is represented in $\mathbf{D}$ by drawing all of their lifts in the closed fundamental domain $F_{1}$ as green line segments. Thus there may be up to eight such segments.

Let $P$ be the set of four points in $S_{1}^{2}$ consisting of the terminal endpoints of the $\beta_{j}$ 's. The data in (3) determines a push-point homeomorphism $h:\left(S_{1}^{2}, P_{1}\right) \rightarrow\left(S_{1}^{2}, P\right)$, unique up to isotopy rel $P_{1}$, by pushing the four points of $P_{1}$ along the (possibly trivial) oriented green geodesics $\beta_{j}$.

The result below follows immediately from straightforward arguments.

Lemma 3.3. The map $f=h \circ g=h \circ \phi \circ \overline{i d}:\left(S_{1}^{2}, P\right) \rightarrow\left(S_{1}^{2}, P\right)$ is a Thurston map; it is a $N E T$ map if $\# P_{f}=4$. Once the data (1), (2), and (3) are fixed, the following are uniquely determined:

(1) the subset $P_{f} \subset P \subset S_{1}^{2}$;

(2) the isotopy class of $f$ relative to $P \subset S_{1}^{2}$;

(3) the isotopy class of the conjugate $F:=\overline{\mathrm{id}} \circ f \circ \overline{\mathrm{id}}^{-1}=\overline{\mathrm{id}} \circ h \circ \phi:\left(S_{2}^{2}, P_{2}\right) \rightarrow\left(S_{2}^{2}, P_{2}\right)$;

$P_{F} \subset P_{2}$ and equality holds iff $F$ (equivalently, $f$ ) is a NET map;

(4) the equivalence class of $f$.

If $\# P_{f}=4$ (equivalently, if $P_{f}=P$ ) then we call the data in (1), (2), (3) above NET map data and we say that (1), (2), (3) give a NET map presentation for $f$. This presentation is encoded in the diagram $\mathbf{D}$.

\section{Remarks:}

(1) The dynamical plane - the sphere $S_{1}^{2}$ - and postcritical set of $f$ depend on the data in (1), (2), (3), while those of its conjugate $F$ do not. The former representation is more convenient for computations of dynamical invariants like slope functions, while the latter is more convenient in other situations since the set of isotopy classes of Thurston maps with a common postcritical set $P_{2}$ is naturally a biset over the pure mapping class group $P \Gamma(2)$.

(2) Suppose a diagram $\mathbf{D}$ is fixed; let $P \Gamma(2) .[\mathbf{D}]$ be the orbit of its projective class under the natural free action of the pure mapping class group $P \Gamma(2)$. Theorem 1.2 implies that the image of the orbit is a subset of isotopy classes of NET maps that naturally forms an irreducible biset $\mathcal{F}$ over the group $P \Gamma(2)$ in the sense of [10]. If some (equivalently, any) map in this orbit is not Euclidean, then the natural map $[\mathbf{D}] \mapsto F$ 


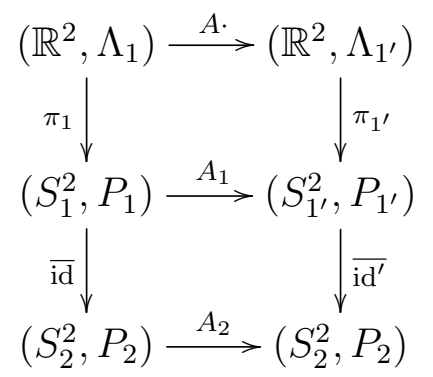

Figure 4. Maps induced by the matrix $A$

is never injective on the level of isotopy classes, since by [6, Theorem 6.3] there will always be elements $A$ for which $A_{2} \circ F=F$ up to isotopy.

\section{AfFine tWisting of Diagrams}

In this section, we prove Theorems 1.2 and 4.4 .

Let $\mathbf{D}$ be a NET map presentation diagram, $f:\left(S_{1}^{2}, P\right) \rightarrow\left(S_{1}^{2}, P\right)$ the corresponding NET map with postcritical set $P$, and $F:\left(S_{2}^{2}, P_{2}\right) \rightarrow\left(S_{2}^{2}, P_{2}\right)$ the corresponding conjugate. Recall from Lemma 3.3 that the isotopy class of $F$ relative to $P_{2}$ is independent of the choice used in the definition of push homeomorphism. Let $A \in \operatorname{Mat}^{+}(2, \mathbb{Z})$, let $\mathbf{D}^{\prime}:=A \cdot \mathbf{D}$ be the NET map presentation diagram obtained by applying the linear map induced by $A$ to the diagram $\mathbf{D}$, and let $F^{\prime}:\left(S_{2}^{2}, P_{2}\right) \rightarrow\left(S_{2}^{2}, P_{2}\right)$ be the corresponding NET map.

Proof of Theorem 1.2. For clarity of exposition, we use primes to distinguish objects associated with $\mathbf{D}^{\prime}$, and, as usual, subscripts 1 and 2 to distinguish between quotients associated to lattices $\Lambda_{1}, \Lambda_{2}$.

We have $\Lambda_{1^{\prime}}=A \cdot \Lambda_{1}$ by the definition of the action, so the diagram in Figure 4 commutes. Hence, abusing notation, $\Phi^{\prime}=A \circ \Phi$ and $\phi^{\prime}=A_{1} \circ \phi$.

We next consider the effect on the push homeomorphism. Again by the definition of the action, $A_{1}$ sends the green $\operatorname{arcs} \beta_{j}$ to the green $\operatorname{arcs} \beta_{j}^{\prime}$. It follows that the push homeomorphisms $h_{1}$ and $h_{1^{\prime}}$ (subscript 1's added for clarity) in the definitions of $f$ and of $f^{\prime}$ may be chosen so that

$$
h_{1^{\prime}}=A_{1} \circ h_{1} \circ A_{1}^{-1} .
$$

Now consider the diagram in Figure 5. The triangle commutes because $\phi^{\prime}=A_{1} \circ \phi$. Line 1 shows that the left square commutes. The bottom square of Figure 4 shows that the right square commutes. Thus the diagram in Figure 5 commutes, and so $F^{\prime}=A_{2} \circ F$.

This provesTheorem 1.2.

The rest of this section is devoted to the analog of Theorem 1.2 for translations. We maintain the notation $\Lambda_{2}, \Gamma_{2}, \pi_{2}, S_{2}^{2}$ and $P_{2}$ of Theorem 1.2 . Now let $b$ be a vector in $\Lambda_{2}=\mathbb{Z}^{2}$. The translation $x \mapsto x+b$ on $\mathbb{R}^{2}$ induces a homeomorphism $b_{2}:\left(S_{2}^{2}, P_{2}\right) \rightarrow\left(S_{2}^{2}, P_{2}\right)$, an element of the modular group of $\left(S_{2}^{2}, P_{2}\right)$. Because $\Gamma_{2}$ contains all translations by elements in $2 \Lambda_{2}$, this homeomorphism depends only on the coset $b+2 \Lambda_{2}$ in $\Lambda_{2} / 2 \Lambda_{2}$. We identify $\Lambda_{2} / 2 \Lambda_{2}$ with $\mathbb{Z}_{2}^{2}$ by means of the canonical group homomorphism from $\mathbb{Z}^{2}$ to $\mathbb{Z}_{2}^{2}$. In this way $b$ determines an element $\bar{b} \in \mathbb{Z}_{2}^{2}$. We obtain another element of $\mathbb{Z}_{2}^{2}$ from a NET map presentation diagram $\mathbf{D}$ as follows. Let $\Lambda_{1}$ be the lattice associated to $\mathbf{D}$ with ordered basis 


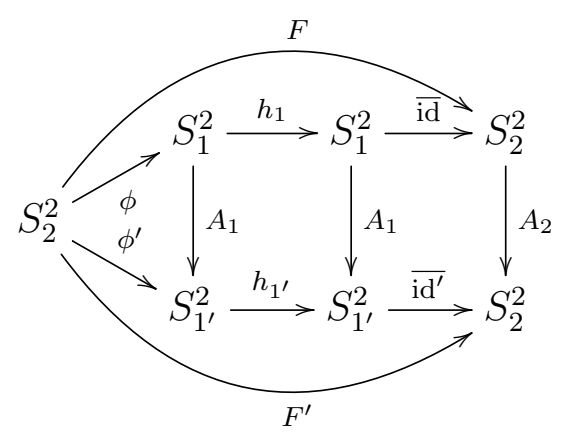

Figure 5. Proving Theorem 1.2

$\left(\lambda_{1}, \lambda_{2}\right)$. One of the lattice points $\left\{0, \lambda_{1}, \lambda_{2}, \lambda_{1}+\lambda_{2}\right\}$ in the boundary of $\mathbf{D}$ is circled. Call it $a$. There is a group homomorphism $\Lambda_{1} \rightarrow \mathbb{Z}_{2}^{2}$ which sends $\lambda_{1}$ to $(1,0)$ and $\lambda_{2}$ to $(0,1)$. Let $\bar{a}$ denote the image in $\mathbb{Z}_{2}^{2}$ of $a$ under this group homomorphism. Now we have an element $\bar{b}$ of $\mathbb{Z}_{2}^{2}$ associated to $b$ and an element $\bar{a}$ of $\mathbb{Z}_{2}^{2}$ associated to D. We obtain a new NET map presentation diagram $\mathbf{D}^{\prime}$ such that $\mathbf{D}^{\prime}$ is the same as $\mathbf{D}$ except that now the translation term corresponding to $\mathbf{D}^{\prime}$ is $\bar{b}+\bar{a}$. The map $\mathbf{D} \mapsto \mathbf{D}^{\prime}$ extends to projective classes $[\mathbf{D}] \mapsto\left[\mathbf{D}^{\prime}\right]$. We have:

Theorem 4.4. Suppose $b \in \mathbb{Z}^{2}$ sends $[\mathbf{D}]$ to $\left[\mathbf{D}^{\prime}\right]$. Let $F, F^{\prime}:\left(S_{2}^{2}, P_{2}\right) \rightarrow\left(S_{2}^{2}, P_{2}\right)$ be the corresponding Thurston maps. Then $F^{\prime}=b_{2} \circ F$, where $b_{2}:\left(S_{2}^{2}, P_{2}\right) \rightarrow\left(S_{2}^{2}, P_{2}\right)$ is the map induced by $b$.

Proof. Let $e_{1}=(1,0)$ and $e_{2}=(0,1)$, the standard basis vectors in $\mathbb{Z}^{2}$. We have a straightforward bijection $\tau:\left\{0, e_{1}, e_{2}, e_{1}+e_{2}\right\} \rightarrow\left\{0, \lambda_{1}, \lambda_{2}, \lambda_{1}+\lambda_{2}\right\}$. The homeomorphism $\phi$ conjugates the element in the modular group of $\left(S_{2}^{2}, P_{2}\right)$ induced by $x \mapsto x+e$, where $e \in\left\{0, e_{1}, e_{2}, e_{1}+e_{2}\right\}$, to the element of the modular group of $\left(S_{1}^{2}, P_{1}\right)$ induced by $x \mapsto x+\tau(e)$. Theorem 4.4 follows easily from this.

\section{Presentation and equivalence of Euclidean NET maps}

Completeness of construction. In this paragraph, we show that the conditions in data items (1) and (2) of $\$ 3$ obtains every Thurston equivalence class of Euclidean NET maps. So, let $g: S^{2} \rightarrow S^{2}$ be a Euclidean NET map. As in Section 1 of [3], we construct lattices $\Lambda_{i}$, groups $\Gamma_{i}$ of Euclidean isometries and quotient maps $\pi_{i}: \mathbb{R}^{2} \rightarrow \mathbb{R}^{2} / \Gamma_{i}=S_{i}^{2}$ for $i \in\{1,2\}$. There exist identifications of $S_{i}^{2}$ with the dynamical plane $S^{2}$ of $g$ so that $\pi_{i}\left(\Lambda_{i}\right)$ is the postcritical set $P_{g}$ of $g$ for $i \in\{1,2\}$ and $g \circ \pi_{1}=\pi_{2}$. We have the right half of Figure 6 , Now we apply statement 2 of Lemma 2.2 with $\Lambda=\Lambda_{2}, \Lambda^{\prime}=\Lambda_{1}$ and $\phi: S^{2} \rightarrow S^{2}$ the identity map. We obtain an affine isomorphism $\Phi: \mathbb{R}^{2} \rightarrow \mathbb{R}^{2}$ such that $\Phi\left(\Lambda_{2}\right)$ is a sublattice of $\Lambda_{1}$ and the branched map which $\Phi$ induces from $S^{2}$ to $S^{2}$ is isotopic to the identity map rel $P_{g}$. Because $\Phi$ induces the identity map, $\Phi\left(\Lambda_{2}\right)=\Lambda_{1}$. Because $g$ has degree greater than 1 , the lattice $\Lambda_{1}$ is a proper sublattice of $\Lambda_{2}$. This proves that the construction is complete.

Equivalence of Euclidean NET map data. Thus far we have a way to construct Euclidean NET maps from our Euclidean NET map data, and we know that every Euclidean NET map has this form. Hence the Thurston equivalence relation on Euclidean NET maps corresponds to an equivalence relation on sets of Euclidean NET map data. Here it is. 


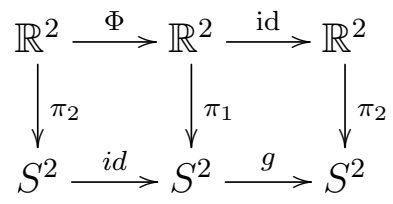

Figure 6. Proving that the construction is complete

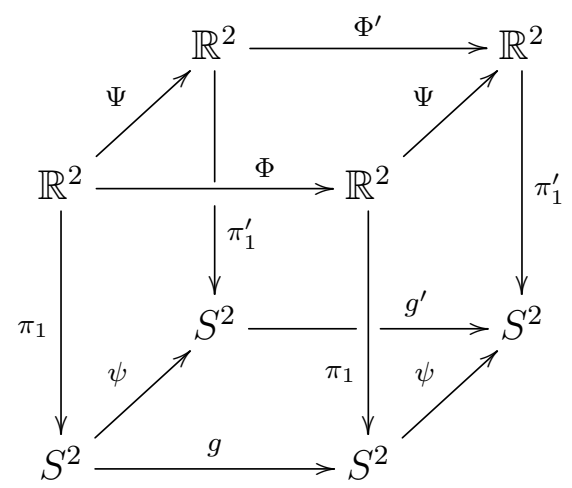

Figure 7. Equivalence of Euclidean NET maps

Equivalence relation on data. Suppose that we have lattices $\Lambda_{2} \subseteq \mathbb{R}^{2}$ and $\Lambda_{2}^{\prime} \subseteq \mathbb{R}^{2}$ and orientation-preserving affine isomorphisms $\Phi: \mathbb{R}^{2} \rightarrow \mathbb{R}^{2}$ and $\Phi^{\prime}: \mathbb{R}^{2} \rightarrow \mathbb{R}^{2}$ such that $\Phi\left(\Lambda_{2}\right)$ is a proper sublattice $\Lambda_{1}$ of $\Lambda_{2}$ and $\Phi^{\prime}\left(\Lambda_{2}^{\prime}\right)$ is a proper sublattice $\Lambda_{1}^{\prime}$ of $\Lambda_{2}^{\prime}$. We say that the pair $\left(\Lambda_{2}, \Phi\right)$ is equivalent to the pair $\left(\Lambda_{2}^{\prime}, \Phi^{\prime}\right)$ if and only if there exists an orientation-preserving affine isomorphism $\Psi: \mathbb{R}^{2} \rightarrow \mathbb{R}^{2}$ such that

(1) $\Psi\left(\Lambda_{2}\right)=\Lambda_{2}^{\prime}$

(2) $\Psi \circ \Phi \circ \Psi^{-1}= \pm \Phi^{\prime}+2 \lambda$ for some $\lambda \in \Lambda_{1}^{\prime}$.

This defines an equivalence relation on data pairs. Using Lemmas 2.1 and 2.2, it is a straightforward exercise to prove that data equivalence implies map equivalence and map equivalence implies data equivalence; see Figure 7.

\section{Preliminary presentations of General NET maps}

In this section we extend the results of the previous section to general NET maps by showing that each NET map $f$ is equivalent to a map presented by what we call preliminary data. This data will factor $f$ as a composition of a Euclidean NET map $g$ and a pushhomeomorphism $h$ where the arcs $\beta_{j}$ along which the pushes occur are pairwise disjoint.

Here is our preliminary presentation for NET maps.

\section{Preliminary NET map data.}

(1) A lattice $\Lambda_{2} \subseteq \mathbb{R}^{2}$.

(2) An orientation-preserving affine isomorphism $\Phi: \mathbb{R}^{2} \rightarrow \mathbb{R}^{2}$ such that $\Phi\left(\Lambda_{2}\right)$ is a proper sublattice $\Lambda_{1}$ of $\Lambda_{2}$.

(3) Four closed arcs $\alpha_{1}, \alpha_{2}, \alpha_{3}, \alpha_{4}$ in $\mathbb{R}^{2}$ such that $\alpha_{i}$ has initial endpoint in $\Lambda_{1}$ and terminal endpoint in $\Lambda_{2}$ for every $i \in\{1,2,3,4\}$. Furthermore, the images of $\alpha_{1}, \alpha_{2}$, 
$\alpha_{3}, \alpha_{4}$ in $\mathbb{R}^{2} / \Gamma_{1}$ are disjoint, where $\Gamma_{1}$ is the group of Euclidean isometries of the form $x \mapsto 2 \lambda \pm x$ for some $\lambda \in \Lambda_{1}$.

In the present case of general NET maps, our preliminary data do not always determine an equivalence class of NET maps, but only because the Thurston maps which we obtain might have fewer than four postcritical points.

Construction. We use the preliminary NET map data to construct an equivalence class of Thurston maps. These maps will be NET maps if they have at least four postcritical points, which is almost always the case. The data in items (1) and (2) constitute Euclidean NET map data. Let $g: S^{2} \rightarrow S^{2}$ be a Euclidean NET map associated to the data in items (1) and (2).

Now we turn our attention to the construction of a homeomorphism $h: S^{2} \rightarrow S^{2}$. The construction of $g$ involves an identification of $\mathbb{R}^{2} / \Gamma_{1}$ with $S^{2}$. So $\alpha_{1}, \alpha_{2}, \alpha_{3}, \alpha_{4}$ map to disjoint closed arcs $\beta_{1}, \beta_{2}, \beta_{3}, \beta_{4}$ in $S^{2}$. We take $h: S^{2} \rightarrow S^{2}$ to be a push map along $\beta_{1}, \beta_{2}$, $\beta_{3}, \beta_{4}$.

Now that $g$ and $h$ are defined, we set $f=h \circ g$. Let $P_{1}$ be the set of initial endpoints of $\beta_{1}, \beta_{2}, \beta_{3}, \beta_{4}$, and let $P$ be the set of terminal endpoints of $\beta_{1}, \beta_{2}, \beta_{3}, \beta_{4}$. The definitions imply that $P_{1}$ is the postcritical set of $g$ and that $g(P) \subseteq P_{1}$. It follows that $f$ is a Thurston map, and it is a NET map if it has at least four postcritical points. In this case, $P$ is the postcritical set of $f$. This completes the construction of a Thurston map $f$ from the preliminary NET map data.

We next show that the equivalence class of the Thurston map $f$ just constructed is independent of the choices involved. There are two choices. The first involves the identification of $\mathbb{R}^{2} / \Gamma_{1}$ with $S^{2}$. Changing this identification corresponds to conjugating $f$ by a homeomorphism. The result is equivalent to $f$. The second choice is the choice of $h$. But $h$ is unique up to isotopy rel $P_{1}$. Hence because $g(P) \subseteq P_{1}$, changing $h$ amounts to changing $f$ by an isotopy rel $P$. This is also equivalent to $f$.

Completeness of construction. We show that this construction obtains every equivalence class of NET maps. So, let $f: S^{2} \rightarrow S^{2}$ be a NET map. We have the usual lattices $\Lambda_{1}$ and $\Lambda_{2}$, groups $\Gamma_{1}$ and $\Gamma_{2}$, maps $\pi_{1}$ and $\pi_{2}$ and four-element sets $P_{1}$ and $P_{2}$ coming from Section 1 of [3]. Lemma 1.3 of [3] shows that there exist exactly four points in $S^{2}$ which are not critical points of $f$ such that $f$ maps them to its postcritical set. We choose an identification of $S^{2}$ with $S_{1}^{2}$ so that $P_{1}$ is this set of four points. We choose an identification of $S^{2}$ with $S_{2}^{2}$ so that $P_{2}$ is the postcritical set of $f$. We construct four disjoint closed arcs $\beta_{1}, \beta_{2}, \beta_{3}$, $\beta_{4}$ in $S^{2}$ such that the initial endpoint of $\beta_{i}$ is in $P_{1}$ and the terminal endpoint of $\beta_{i}$ is in $P_{2}$ for every $i \in\{1,2,3,4\}$. We choose four closed arcs $\alpha_{1}, \alpha_{2}, \alpha_{3}, \alpha_{4}$ in $\mathbb{R}^{2}$ such that $\pi_{1}$ maps $\alpha_{i}$ homeomorphically to $\beta_{i}$ for every $i \in\{1,2,3,4\}$. Then the initial endpoint of $\alpha_{i}$ is in $\Lambda_{1}$ and the terminal endpoint of $\alpha_{i}$ is in $\Lambda_{2}$ for every $i \in\{1,2,3,4\}$. Hence we have the NET map data in item (3). Let $h: S^{2} \rightarrow S^{2}$ be a push map relative to $\beta_{1}, \beta_{2}, \beta_{3}, \beta_{4}$. We know that then $f=h \circ g$, where $g$ is a Euclidean NET map. Section 5 shows that $g$ is Thurston equivalent to a Euclidean NET map $g^{\prime}$ which arises from NET map data as in items (1) and (2). This means that there exist homeomorphisms $\psi_{1}: S^{2} \rightarrow S^{2}$ and $\psi_{2}: S^{2} \rightarrow S^{2}$ which are isotopic rel $P_{1}$ such that $g^{\prime} \circ \psi_{2}=\psi_{1} \circ g$. Let $h^{\prime}=\psi_{2} \circ h \circ \psi_{1}^{-1}$, and let $f^{\prime}=h^{\prime} \circ g^{\prime}$. We have the commutative diagram in Figure 8. We see that $f$ and $f^{\prime}$ are conjugate, hence Thurston equivalent. Because $\psi_{1}$ and $\psi_{2}$ are isotopic rel $P_{1}=P_{g}$, the maps $\psi_{1}^{-1}$ and $\psi_{2}^{-1}$ are isotopic rel $P_{g^{\prime}}$. So $h^{\prime}$ is isotopic rel $P_{g^{\prime}}$ to $h^{\prime \prime}=\psi_{2} \circ h \circ \psi_{2}^{-1}$, which is clearly a push map of the 


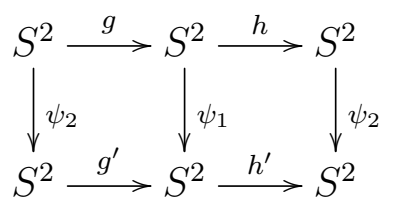

Figure 8. Proving completeness of the construction

desired form. So $f$ is Thurston equivalent to a NET map $f^{\prime \prime}=h^{\prime \prime} \circ g^{\prime}$ which arises from NET map data. This shows that our construction obtains every equivalence class of NET maps.

Conjugation equivalence of NET map data. Just as for Euclidean NET map data, the Thurston equivalence relation on NET maps corresponds to an equivalence relation on preliminary NET map data. We are about to define a conjugation equivalence relation on preliminary NET map data analogous to the equivalence relation on Euclidean NET map data. Unlike the Euclidean case, this conjugation equivalence relation is in general strictly stronger than the equivalence relation on preliminary NET map data which corresponds to the Thurston equivalence relation on NET maps.

Conjugation equivalence relation on preliminary data. Suppose that we have two sets of NET map data. So we have lattices $\Lambda_{2} \subseteq \mathbb{R}^{2}$ and $\Lambda_{2}^{\prime} \subseteq \mathbb{R}^{2}$ and orientation-preserving affine isomorphisms $\Phi: \mathbb{R}^{2} \rightarrow \mathbb{R}^{2}$ and $\Phi^{\prime}: \mathbb{R}^{2} \rightarrow \mathbb{R}^{2}$ such that $\Phi\left(\Lambda_{2}\right)$ is a proper sublattice $\Lambda_{1}$ of $\Lambda_{2}$ and $\Phi^{\prime}\left(\Lambda_{2}^{\prime}\right)$ is a proper sublattice $\Lambda_{1}^{\prime}$ of $\Lambda_{2}^{\prime}$. We also have closed $\operatorname{arcs} \alpha_{1}, \alpha_{2}, \alpha_{3}$, $\alpha_{4}$ in $\mathbb{R}^{2}$ such that $\alpha_{i}$ has initial endpoint in $\Lambda_{1}$ and terminal endpoint in $\Lambda_{2}$ and closed $\operatorname{arcs} \alpha_{1}^{\prime}, \alpha_{2}^{\prime}, \alpha_{3}^{\prime}, \alpha_{4}^{\prime}$ in $\mathbb{R}^{2}$ such that $\alpha_{i}^{\prime}$ has initial endpoint in $\Lambda_{1}^{\prime}$ and terminal endpoint in $\Lambda_{2}^{\prime}$. Furthermore, the images $\beta_{1}, \beta_{2}, \beta_{3}, \beta_{4}$ of $\alpha_{1}, \alpha_{2}, \alpha_{3}, \alpha_{4}$ in $\mathbb{R}^{2} / \Gamma_{1}$ are disjoint, and the images $\beta_{1}^{\prime}, \beta_{2}^{\prime}, \beta_{3}^{\prime}, \beta_{4}^{\prime}$ of $\alpha_{1}^{\prime}, \alpha_{2}^{\prime}, \alpha_{3}^{\prime}, \alpha_{4}^{\prime}$ in $\mathbb{R}^{2} / \Gamma_{1}^{\prime}$ are disjoint. We say that the triple $\left(\Lambda_{2}, \Phi,\left\{\alpha_{1}, \alpha_{2}, \alpha_{3}, \alpha_{4}\right\}\right)$ is conjugation equivalent to the triple $\left(\Lambda_{2}^{\prime}, \Phi^{\prime},\left\{\alpha_{1}^{\prime}, \alpha_{2}^{\prime}, \alpha_{3}^{\prime}, \alpha_{4}^{\prime}\right\}\right)$ if and only if there exists an orientation-preserving affine isomorphism $\Psi: \mathbb{R}^{2} \rightarrow \mathbb{R}^{2}$ such that

(1) $\Psi\left(\Lambda_{2}\right)=\Lambda_{2}^{\prime}$

(2) $\Psi \circ \Phi \circ \Psi^{-1}= \pm \Phi^{\prime}+2 \lambda$ for some $\lambda \in \Lambda_{1}^{\prime}$;

(3) There is an isotopy of $\mathbb{R}^{2} / \Gamma_{1}^{\prime}$ rel $P_{1}^{\prime} \cup P_{2}^{\prime}$ taking $\psi \circ \beta_{1}, \psi \circ \beta_{2}, \psi \circ \beta_{3}, \psi \circ \beta_{4}$ to $\beta_{1}^{\prime}, \beta_{2}^{\prime}$, $\beta_{3}^{\prime}, \beta_{4}^{\prime}$, not necessarily in order, where $\psi: \mathbb{R}^{2} / \Gamma_{1} \rightarrow \mathbb{R}^{2} / \Gamma_{1}^{\prime}$ is the map of Lemma 2.1 induced by $\Psi$.

This defines an equivalence relation on preliminary data triples. One can show as in Section 5 that if two sets of preliminary data are conjugation equivalent, then the corresponding NET maps are equivalent.

\section{THE EFFECT OF TWISTING ON PRELIMINARY DATA}

Suppose $f$ is a NET map, and suppose that we have expressed $f$ in terms of preliminary data as in the previous subsection, so that $f$ factors as $f=h \circ g$. The arcs $\beta_{j}$ along which the push-homeomorphism $h$ occurs may be very wild. In Section 8 we will show that we can absorb their complexity into the affine part $g$. To do this we now examine the effects of twisting on preliminary data. This result is closely related to Theorems 1.2 and 4.4 .

Theorem 7.1. Let $f$ be a NET map. We construct lattices and maps $\Lambda_{1}, \Lambda_{2}, \pi_{1}, \pi_{2}$ as in the last section's proof of the completeness of the construction. Relative to these maps $f$ decomposes as $f=h \circ g$, where $g$ is a Euclidean NET map and $h$ is a homeomorphism. 


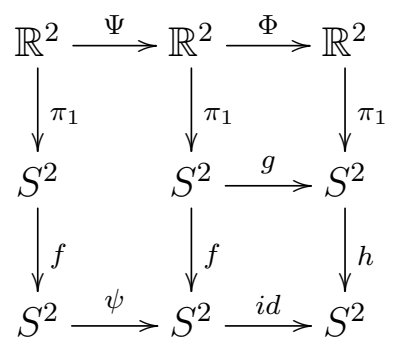

Figure 9. Proving Theorem 7.1

Suppose that $g$ lifts via $\pi_{1}$ to an affine isomorphism $\Phi: \mathbb{R}^{2} \rightarrow \mathbb{R}^{2}$ such that $\Phi\left(\Lambda_{2}\right)=\Lambda_{1}$. Let $\psi:\left(S^{2}, P\right) \rightarrow\left(S^{2}, P\right)$ be an orientation-preserving branched covering map which lifts via $\pi_{2}$ to an affine isomorphism $\Psi: \mathbb{R}^{2} \rightarrow \mathbb{R}^{2}$ such that $\Psi\left(\Lambda_{2}\right) \subseteq \Lambda_{2}$. Then $\psi \circ f=h \circ g^{\prime}$, where $g^{\prime}$ is the Euclidean NET map which lifts via $\pi_{1}$ to $\Phi \circ \Psi$.

Proof. As is usual for NET maps, we have that $\pi_{2}=f \circ \pi_{1}$. So since $\psi$ lifts to $\Psi$ via $\pi_{2}$, we have the left half of the commutative diagram in Figure 9. The rest of Figure 9 results from the facts that $f=h \circ g$ and that $g$ lifts to $\Phi$ via $\pi_{1}$. It need not be true that $\Psi\left(\Lambda_{1}\right)=\Lambda_{1}$, so there need not be an induced homeomorphism from $S^{2}$ to $S^{2}$ subdividing the left half of Figure 9 into two squares. However, $(\Phi \circ \Psi)\left(\Lambda_{1}\right) \subseteq(\Phi \circ \Psi)\left(\Lambda_{2}\right) \subseteq \Phi\left(\Lambda_{2}\right)=\Lambda_{1}$, and so Lemma 2.1 with $\Lambda=\Lambda^{\prime}=\Lambda_{1}$ implies that $\Phi \circ \Psi$ induces a branched covering map $g^{\prime}: S^{2} \rightarrow S^{2}$ via $\pi_{1}$. This and the surjectivity of $\pi_{1}$ imply that $\psi \circ f=h \circ g^{\prime}$.

This proves Theorem 7.1 .

Remark. One might find it unsettling that the left action of $\psi$ on $f$ in Theorem 7.1 seems to correspond to a right action of $\Psi$ on $\Phi$. However, keep in mind that $\Phi$ lifts $g$ via $\pi_{1}$ and that $\Psi$ lifts $\psi$ via $\pi_{2}$. While $\pi_{1}$ does not change in passing from $f$ to $\psi \circ f$, the map $\pi_{2}$ does change. Hence there is no right action here.

\section{NET MAP PRESENTATIONS}

In this section we prove Theorem 1.1 .

Recall that in Section 3 we defined a NET map presentation to consist of three things. The following optional condition can also be imposed if desired.

(4) There exist integers $m \geq 2$ and $n \geq 1$ such that i) $n$ divides $m$, ii) $m$ divides $\lambda_{1}$, iii) $n$ divides $\lambda_{2}$ and iv) $\operatorname{det}(A)=m n$.

The integers $m$ and $n$ are the elementary divisors of the integer matrix $A:=\left[\lambda_{1}, \lambda_{2}\right]$. Corollary 5.2 of [8] shows that they are uniquely determined not only by the Thurston equivalence class of a NET map, but even by its modular group Hurwitz class. We use the convention $n \mid m$ instead of the more common condition $m \mid n$ so that pictures of associated fundamental domains tend to be wider than they are tall and so are more convenient to draw.

Having now an ordered basis $\left(\lambda_{1}, \lambda_{2}\right)$, we define an associated fundamental domain for $\Gamma_{1}$ as the parallelogram with corners $0,2 \lambda_{1}, \lambda_{2}$ and $2 \lambda_{1}+\lambda_{2}$.

We are now prepared to prove Theorem 1.1 . 
Proof of Theorem 1.1. As a slight strengthening of Theorem 1.1, we prove that data item (4) can be satisfied in addition to (1), (2) and (3). The strategy of the proof is to define first approximations of the objects in items (1) through (4). These first approximations will be denoted by letters with prime superscripts. The final desired objects will eventually be gotten from these by applying a linear transformation.

Let $f$ be a NET map. Section 6 shows that $f$ is Thurston equivalent to a NET map which arises from the preliminary NET map data presented there. It is always possible to take the lattice $\Lambda_{2}$ in preliminary NET map data item (1) to be $\mathbb{Z}^{2}$. After making this normalization, let $\Phi(x)=A^{\prime} x+b^{\prime}$ be the affine isomorphism in preliminary NET map data item (2). Since $\operatorname{det}\left(A^{\prime}\right)=\operatorname{deg}(f) \geq 2$, there exist integers $m \geq 2$ and $n \geq 1$ with $n \mid m$ and elements $P, Q \in \mathrm{SL}(2, \mathbb{Z})$ such that $A^{\prime}=P\left[\begin{array}{cc}m & 0 \\ 0 & n\end{array}\right] Q$. Now we use the fact from Section 6 that the Thurston equivalence class of $f$ is invariant under conjugation to replace $A^{\prime}$ by $Q^{\prime} Q^{-1}$. As a result, we may assume that $Q=1$. So the first column $\lambda_{1}^{\prime}$ of $A^{\prime}$ is divisible by $m$, and the second column $\lambda_{2}^{\prime}$ of $A^{\prime}$ is divisible by $n$. We have the data in NET map data item (4). Let $\Lambda_{1}^{\prime}$ be the sublattice of $\Lambda_{2}$ generated by $\lambda_{1}^{\prime}$ and $\lambda_{2}^{\prime}$. The lattice $\Lambda_{1}^{\prime}$ will lead to the desired lattice $\Lambda_{1}$, and the vectors $\lambda_{1}^{\prime}$ and $\lambda_{2}^{\prime}$ will lead to the vectors $\lambda_{1}$ and $\lambda_{2}$ in NET map data item (3).

We next define four line segments $\alpha_{1}^{\prime}, \alpha_{2}^{\prime}, \alpha_{3}^{\prime}, \alpha_{4}^{\prime}$ which will lead to the line segments in NET map data item (3). Let $F_{1}^{\prime}$ be the parallelogram in $\mathbb{R}^{2}$ with corners $0,2 \lambda_{1}^{\prime}, \lambda_{2}^{\prime}$ and $2 \lambda_{1}^{\prime}+\lambda_{2}^{\prime}$. Then $F_{1}^{\prime}$ is a fundamental domain for the usual group $\Gamma_{1}^{\prime}$. The interior of $F_{1}^{\prime}$ maps homeomorphically into $\mathbb{R}^{2} / \Gamma_{1}^{\prime}$. We refer to the line segment with endpoints 0 and $2 \lambda_{1}^{\prime}$ as the bottom of $F_{1}^{\prime}$. We refer to the line segment with endpoints $\lambda_{2}^{\prime}$ and $2 \lambda_{1}^{\prime}+\lambda_{2}^{\prime}$ as the top of $F_{1}^{\prime}$. Similarly, the line segment with endpoints 0 and $\lambda_{2}^{\prime}$, respectively $2 \lambda_{1}^{\prime}$ and $2 \lambda_{1}^{\prime}+\lambda_{2}^{\prime}$, is the left, respectively right, side of $F_{1}^{\prime}$. The rotation $x \mapsto 2 \lambda_{1}^{\prime}-x$ identifies the two halves of the bottom of $F_{1}^{\prime}$, and the rotation $x \mapsto 2 \lambda_{1}^{\prime}+2 \lambda_{2}^{\prime}-x$ identifies the two halves of the top of $F_{1}^{\prime}$. The translation $x \mapsto 2 \lambda_{1}^{\prime}+x$ identifies the two sides of $F_{1}^{\prime}$. So the union of the interior of $F_{1}^{\prime}$ and the "left half" of the boundary of $F_{1}^{\prime}$ maps bijectively to $\mathbb{R}^{2} / \Gamma_{1}^{\prime}$. Let $P_{2}$ be the postcritical set of $f$, and let $\widetilde{P}_{2}$ be the inverse image of $P_{2}$ in this union. So $\widetilde{P}_{2}$ consists of four points which are either in the interior of $F_{1}^{\prime}$ or in the left half of its boundary.

We begin to choose four line segments $\alpha_{1}^{\prime}, \alpha_{2}^{\prime}, \alpha_{3}^{\prime}, \alpha_{4}^{\prime}$. If $\widetilde{P}_{2}$ contains an element in the bottom of $F_{1}^{\prime}$, then we choose the element of $\widetilde{P}_{2}$ in the bottom of $F_{1}^{\prime}$ which is nearest 0 , and we take one of our line segments to be the line segment joining 0 and this point. If $\widetilde{P}_{2}$ contains another element in the bottom of $F_{1}^{\prime}$, then we choose the one nearest $\lambda_{1}^{\prime}$, and we take one of our line segments to be the line segment joining $\lambda_{1}^{\prime}$ and this point. If $\widetilde{P}_{2}$ contains an element in the top of $F_{1}^{\prime}$, then we choose line segments analogously using $\lambda_{2}^{\prime}$ and $\lambda_{1}^{\prime}+\lambda_{2}^{\prime}$ instead of 0 and $\lambda_{1}^{\prime}$.

Suppose that 0 is not yet in a line segment. Then we choose an element of $\widetilde{P}_{2}$ not yet chosen with minimal $\lambda_{2}^{\prime}$-coordinate relative to the basis of $\mathbb{R}^{2}$ consisting of $\lambda_{1}^{\prime}$ and $\lambda_{2}^{\prime}$. We take the line segment joining this point and 0 . Similarly, if $\lambda_{1}^{\prime}$ is not yet in a line segment, then we choose an element of $\widetilde{P}_{2}$ not yet chosen with minimal $\lambda_{2}^{\prime}$-coordinate, and we take the line segment joining this point and $\lambda_{1}^{\prime}$. The two line segments chosen thus far in this paragraph might meet. If so, then these two line segments are the diagonals of a (possibly degenerate) quadrilateral, and we simply exchange them for two opposite sides of that quadrilateral. We choose line segments for $\lambda_{2}^{\prime}$ and $\lambda_{1}^{\prime}+\lambda_{2}^{\prime}$ in the same way as for 0 and $\lambda_{1}^{\prime}$, using maximal $\lambda_{2}^{\prime}$-coordinates instead of minimal $\lambda_{2}^{\prime}$-coordinates. 
We identify $\mathbb{R}^{2} / \Gamma_{1}^{\prime}$ and $\mathbb{R}^{2} / \Gamma_{2}$ with $S^{2}$ as usual. Although the instructions for choosing $\alpha_{1}^{\prime}, \alpha_{2}^{\prime}, \alpha_{3}^{\prime}, \alpha_{4}^{\prime}$ are somewhat verbose, it is easy to see that the images $\beta_{1}^{\prime}, \beta_{2}^{\prime}, \beta_{3}^{\prime}, \beta_{4}^{\prime}$ in $S^{2}$ of $\alpha_{1}^{\prime}, \alpha_{2}^{\prime}, \alpha_{3}^{\prime}, \alpha_{4}^{\prime}$ are disjoint arcs. The arcs $\beta_{i}^{\prime}$ are like those in NET map data item (3). Let $h^{\prime}: S^{2} \rightarrow S^{2}$ be a push map relative to $\beta_{1}^{\prime}, \beta_{2}^{\prime}, \beta_{3}^{\prime}, \beta_{4}^{\prime}$ as in Section 6 .

We have that $f=h \circ g$, where $h$ is a push map and $g$ is the Euclidean NET map determined by $\Phi$ and the usual branched covering map $\pi_{1}^{\prime}: \mathbb{R}^{2} \rightarrow S^{2}$ corresponding to $\Gamma_{1}^{\prime}$. So $f=\psi \circ\left(h^{\prime} \circ g\right)$, where $\psi=h \circ h^{\prime-1}$ is an orientation-preserving homeomorphism which stabilizes the postcritical set $P_{2}$ of $f$. Statement 2 of Lemma 2.2 with $\Lambda=\Lambda^{\prime}=\Lambda_{2}=\mathbb{Z}^{2}$ easily implies that there exists an affine isomorphism $\Psi: \mathbb{R}^{2} \rightarrow \mathbb{R}^{2}$ such that $\Psi\left(\Lambda_{2}\right)=\Lambda_{2}$ and $\Psi$ induces by means of the usual branched covering map $\pi_{2}$ a homeomorphism from $S^{2}$ to $S^{2}$ which is isotopic to $\psi$ rel $P_{2}$. After modifying $f$ by an isotopy, we may assume that $\Psi$ induces $\psi$. Suppose that $\Psi(x)=C x+d$.

Now we apply Theorems 1.2 and 4.4 . We conclude that $f$ is Thurston equivalent to a NET map arising from NET map data with the following form. The lattice $\Lambda_{2}$ is preserved. The orientation-preserving affine isomorphism has linear term $A=C A^{\prime}$. The associated green line segments have the form $\alpha_{i}=C \cdot \alpha_{i}^{\prime}$. We take $\lambda_{1}$ and $\lambda_{2}$ to be the columns of $A$. Because $\lambda_{1}^{\prime}$ is divisible by $m$ and $\lambda_{2}^{\prime}$ is divisible by $n$, the corresponding statements are true for $\lambda_{1}$ and $\lambda_{2}$.

It easily follows that we now have a NET map presentation for our original NET map and even that data item (4) is satisfied. We obtain our diagram from this as discussed in Section 3 ,

This proves Theorem 1.1 .

\section{AN ALGORITHM FOR COMPUTING NET MAP PRESENTATIONS}

The definition of a NET map is simple - it is easy to check whether or not a Thurston map is a NET map. The presentation of a NET map is also easy to understand. But finding a presentation of a given NET map can be challenging. The goal of this section is to provide an algorithm which produces a presentation of a given NET map based on topological knowledge of its action on the 2-sphere.

We begin with a discussion of Euclidean NET maps. Let $g$ be a Euclidean NET map. Suppose that $g$ has a NET map presentation with matrix $A$. The translation term $b$ is irrelevant for this discussion, and, of course, its green line segments are all trivial.

We wish to determine $A$ in terms of the action of $g$ on the 2-sphere $S^{2}$. Let $\lambda_{1}$ and $\lambda_{2}$ be the columns of $A$, as usual. Let $e_{1}$ and $e_{2}$ be the standard basis vectors of $\mathbb{Z}^{2}$. Since $A$ expresses $\lambda_{1}$ and $\lambda_{2}$ in terms of $e_{1}$ and $e_{2}$, the matrix $A^{-1}$ expresses $e_{1}$ and $e_{2}$ in terms of $\lambda_{1}$ and $\lambda_{2}$.

Our next goal is to interpret the last statement in terms of simple closed curves in $S^{2}$. We have a branched covering map $\pi_{1}: \mathbb{R}^{2} \rightarrow S^{2}$ as usual taking $0, \lambda_{1}, \lambda_{2}, \lambda_{1}+\lambda_{2}$ onto the postcritical set $P_{g}$ of $g$. Now we compute slopes of simple closed curves in $S^{2} \backslash P_{g}$ using the ordered basis $\left(\lambda_{1}, \lambda_{2}\right)$ rather than $\left(e_{1}, e_{2}\right)$. Hence a simple closed curve in $S^{2} \backslash P_{g}$ with core arc being the image under $\pi_{1}$ of the line segment joining 0 and $\lambda_{1}$ has slope 0 . Similarly, a simple closed curve in $S^{2} \backslash P_{g}$ with core arc being the image under $\pi_{1}$ of the line segment joining 0 and $\lambda_{2}$ has slope $\infty$.

Let $\gamma \subseteq S^{2} \backslash P_{g}$ be a simple closed curve with slope 0 . The slopes of the connected components of $g^{-1}(\gamma)$ are equal. Suppose that this slope is $\frac{p}{q}$, where $p$ and $q$ are relatively 


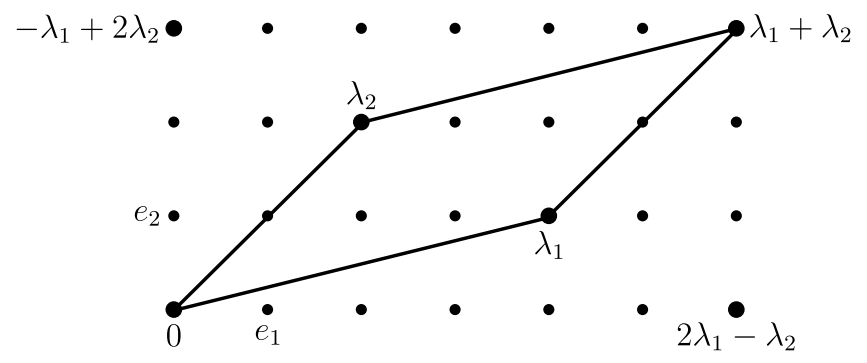

Figure 10. Computing a matrix of a Euclidean NET map

prime integers. This implies that the vector $q \lambda_{1}+p \lambda_{2}$ has the same direction as $\pm e_{1}$. See Figure 10, where $p=-1$ and $q=2$. Because $q \lambda_{1}+p \lambda_{2}$ has integer coordinates, there is a positive integer $d$ such that $\pm d e_{1}=q \lambda_{1}+p \lambda_{2}$. As in Theorem 4.1 of [3], the integer $d$ is the degree with which $g$ maps every connected component of $g^{-1}(\gamma)$ to $\gamma$. Similarly, if $\delta$ is a simple closed curve in $S^{2} \backslash P_{g}$ with slope $\infty$, then $\pm e e_{2}=s \lambda_{1}+r \lambda_{2}$, where every connected component of $g^{-1}(\delta)$ has slope $\frac{r}{s}$ and $g$ maps each of these connected components to $\delta$ with degree $e$. If necessary, we multiply one of the columns of the matrix $\left[\begin{array}{l}q / d s / e \\ p / d\end{array}\right]$ by -1 so that the resulting determinant is positive. Because the determinant of $A$ is positive, the resulting matrix is $\pm A^{-1}$. Thus in this way we are able to compute $A$ up to multiplication by \pm 1 , which is what we need.

For example, for Figure 10, we have that $p=-1, q=2$ and $d=6$. Similarly, $r=2$, $s=-1$ and $e=3$. Checking positivity of the determinant, we find that we may take

$$
A=\left[\begin{array}{cc}
\frac{q}{d} & \frac{s}{e} \\
\frac{p}{d} & \frac{r}{e}
\end{array}\right]^{-1}=\left[\begin{array}{cc}
\frac{1}{3} & \frac{-1}{3} \\
\frac{-1}{6} & \frac{2}{3}
\end{array}\right]^{-1}=6\left[\begin{array}{cc}
\frac{2}{3} & \frac{1}{3} \\
\frac{1}{6} & \frac{1}{3}
\end{array}\right]=\left[\begin{array}{ll}
4 & 2 \\
1 & 2
\end{array}\right],
$$

in agreement with the facts that $\lambda_{1}=(4,1)$ and $\lambda_{2}=(2,2)$.

Before describing the algorithm, we make a remark regarding notation. The above proof of Theorem 1.1 begins by defining objects denoted by primed characters. In general, these objects must be modified by a linear transformation to obtain the desired objects denoted by unprimed characters. In practice this linear transformation is unnecessary. So in our description of the algorithm, we reverse the notation; we begin with unprimed characters and end with primed characters.

We are now ready to present an algorithm which finds a presentation for a general NET map $f$. Here it is.

Step 1. Identify the postcritical set $P_{2}$ of $f$.

Step 2. Identify the set $P_{1}$ of four points in $f^{-1}\left(P_{2}\right)$ which are not critical points.

Lemma 1.3 of [3] shows that $P_{1}$ exists.

Step 3. Construct four disjoint (green) $\operatorname{arcs} \beta_{1}, \beta_{2}, \beta_{3}, \beta_{4}$ in $S^{2}$ each with one endpoint in $P_{1}$ and one endpoint in $P_{2}$.

These arcs determine a push map $h$ up to isotopy rel $P_{1}$ such that $h\left(P_{1}\right)=P_{2}$. Set $g=h^{-1} \circ f$. Then, as in Theorem 2.1 of [3], $g$ is a Euclidean NET map with postcritical set $P_{1}$. 


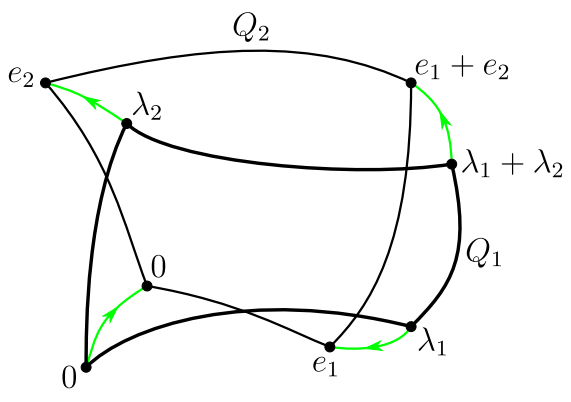

Figure 11. The quadrilaterals $Q_{1}$ and $Q_{2}$

Step 4. Construct a simple closed curve in $S^{2}$ containing $P_{1}$ which meets every $\beta_{i}$ in at most its endpoints. Label the points of $P_{1}$ with labels $\mathbf{0}, \lambda_{1}, \lambda_{1}+\lambda_{2}, \lambda_{2}$ in cyclic order around the curve. This curve together with this labeling of these four points determines a topological quadrilateral, $Q_{1}$, so that the orientation of the labeled points is counterclockwise relative to $Q_{1}$.

See Figure 11, where $\beta_{1}, \beta_{2}, \beta_{3}, \beta_{4}$ are drawn as green arcs. Arrows on them indicate push directions. One of the cyclic labelings of $Q_{1}$ is shown. (The elements of $P_{1}$ will be the images of lattice points $0, \lambda_{1}, \lambda_{1}+\lambda_{2}$ and $\lambda_{2}$ in $\mathbb{R}^{2}$ under the usual branched covering map $\pi_{1}: \mathbb{R}^{2} \rightarrow S^{2}$. We view $Q_{1}$ as the image of the parallelogram in Figure 10 under $\pi_{1}$.)

Step 5. (Optional) Construct $g^{-1}\left(\partial Q_{1}\right)$ up to isotopy rel $P_{1}$.

Although this step is optional, it is useful when performing Step 6 and when constructing the covering map $\pi_{1}$ between Steps 8 and 9 . We may view $g^{-1}\left(\partial Q_{1}\right)$ as a 1-complex in $S^{2}$ with vertex set $g^{-1}\left(P_{1}\right)$. The elements of $P_{1}$ are vertices of $g^{-1}\left(\partial Q_{1}\right)$ with valence 2 . All other vertices have valence 4 .

Now we use $Q_{1}$ to define slopes of simple closed curves in $S^{2} \backslash P_{1}$. The edge of $Q_{1}$ with labels 0 and $\lambda_{1}$ corresponds to slope 0 , and the edge of $Q_{1}$ with labels 0 and $\lambda_{2}$ corresponds to slope $\infty$. (We are computing slopes here using the ordered basis $\left(\lambda_{1}, \lambda_{2}\right)$ of $\mathbb{R}^{2}$, not the standard ordered basis $\left(e_{1}, e_{2}\right)$.) Let $\gamma$ and $\delta$ be simple closed curves in $S^{2} \backslash P_{1}$ with slopes 0 and $\infty$, respectively.

Step 6. Compute the slope $\frac{p}{q}$ in reduced form of one component of $g^{-1}(\gamma)$, and compute the degree $d$ with which $g$ maps this component to $\gamma$. Compute the slope $\frac{r}{s}$ in reduced form of one component of $g^{-1}(\delta)$, and compute the degree $e$ with which $g$ maps this component to $\delta$.

The 1-complex $g^{-1}\left(\partial Q_{1}\right)$ should be helpful here.

Step 7. Multiply one column of the matrix $\left[\begin{array}{ll}q / d & s / e \\ p / d & r / e\end{array}\right]$ by -1 if necessary so that the result has positive determinant. Compute the inverse $A$ of this matrix.

The discussion at the beginning of this section shows that the matrix $A$ is a matrix of integers which is a presentation matrix for $g$.

Step 8. Let $x$ be the element of $P_{1}$ with label 0 , and determine the label $b$ of $g(x)$.

Let $\lambda_{1}$ and $\lambda_{2}$ be the columns of $A$. We have an affine automorphism $\Phi(x)=A x+b$ of $\mathbb{R}^{2}$. Now we define our usual branched covering map $\pi_{1}: \mathbb{R}^{2} \rightarrow S^{2}$ so that $\pi_{1}(\lambda)$ is the element 
of $P_{1}$ with label $\lambda$ for $\lambda \in\left\{0, \lambda_{1}, \lambda_{2}, \lambda_{1}+\lambda_{2}\right\}$ and $\pi_{1}$ takes the 1-skeleton of the standard tiling of $\mathbb{R}^{2}$ by squares to $g^{-1}\left(\partial Q_{1}\right)$, at least up to isotopy rel $\mathbb{Z}^{2}$. The lifts of $Q_{1}$ to $\mathbb{R}^{2}$ yield fundamental domains for $\Lambda_{1}$ and the lifts of $g^{-1}\left(Q_{1}\right)$ yield fundamental domains for $\Lambda_{2}$. The parallelogram $F_{1}$ in $\mathbb{R}^{2}$ with corners $0,2 \lambda_{1}, \lambda_{2}$ and $2 \lambda_{1}+\lambda_{2}$ is a fundamental domain for the usual group $\Gamma_{1}$.

Step 9. Construct line segments $\alpha_{i}$ in $F_{1}$ such that the arcs $\pi_{1}\left(\alpha_{i}\right)$ form a set of four disjoint arcs, each with one endpoint in $P_{1}$ and one endpoint in $P_{2}$.

If a homotopy of $S^{2}$ rel $P_{1} \cup P_{2}$ takes the union of the $\operatorname{arcs} \pi_{1}\left(\alpha_{i}\right)$ to the union of the arcs $\beta_{i}$, then we are done: we have an affine isomorphism $\Phi(x)=A x+b$, a parallelogram which is a fundamental domain $F_{1}$ for $\Gamma_{1}$ and appropriate green line segments $\alpha_{i}$.

Otherwise, we continue. What follows is essentially an algorithm for computing a NET map presentation of a twist of a NET map with known presentation. Let $k: S^{2} \rightarrow S^{2}$ be a push map relative to the images of the $\alpha_{i}$ 's in $S^{2}$ such that $k\left(P_{1}\right)=P_{2}$. Then $f=h \circ g=$ $\psi \circ(k \circ g)$, where $\psi=h \circ k^{-1}$ is an orientation-preserving homeomorphism which stabilizes $P_{2}$. We have a presentation of the desired form for the NET map $k \circ g$. We wish to transform this presentation to a presentation for $f$, a modular group twist of $k \circ g$. This leads us to seek an affine isomorphism $\Psi: \mathbb{R}^{2} \rightarrow \mathbb{R}^{2}$ which induces $\psi$ relative to the branched covering map $\pi_{2}=f \circ \pi_{1}$. For this we work with simple closed curves in $S^{2} \backslash P_{2}$, calculating their slopes using a quadrilateral $Q_{2}$ isotopic to $h\left(Q_{1}\right)$ rel $P_{2}$ just as we used $Q_{1}$ to calculate slopes of simple closed curves in $S^{2} \backslash P_{1}$. See Figure 11. Now we take $\gamma$ and $\delta$ to be simple closed curves in $S^{2} \backslash P_{2}$ with slopes 0 and $\infty$, respectively.

Step 10. Compute the slopes $\frac{p}{q}$ and $\frac{r}{s}$ in reduced form of $\psi(\gamma)$ and $\psi(\delta)$.

Step 11. Multiply one column of the matrix $\left[\begin{array}{cc}q & s \\ p & r\end{array}\right]$ by -1 if necessary so that the result has determinant 1 . Let $C$ be the resulting matrix.

Step 12. Label the vertices of $Q_{2}$ with labels $0, e_{1}, e_{1}+e_{2}, e_{2}$ consistently with the labels of $Q_{1}$. Let $x$ be the element of $P_{2}$ with label 0 , and let $d$ be the label of $\psi(x)$.

Then the affine automorphism $\Psi(x)=C x+d$ of $\mathbb{R}^{2}$ induces $\psi$ relative to $\pi_{2}$. Theorems 1.2 and 4.4 now show how to transform the presentation diagram of $k \circ g$ to obtain a presentation diagram for $f$.

Step 13. Compute the matrix $A^{\prime}=C A$, vector $b^{\prime} \in\left\{0, C \lambda_{1}, C \lambda_{2}, C\left(\lambda_{1}+\lambda_{2}\right)\right\}$ congruent to $C A d+C b$ modulo $2\left\langle C \lambda_{1}, C \lambda_{2}\right\rangle$, fundamental domain $F_{1}^{\prime}=C F_{1}$ and line segments $\alpha_{i}^{\prime}=C \alpha_{i}$.

These primed quantities provide our presentation for $f$.

Example 9.1. We illustrate the above algorithm by deriving the NET map presentation for the rabbit $f(z)=z^{2}+c_{R}$ in Figure 1. Figure 12 is a diagram of a finite subdivision rule for $f$. The initial cell structure on $S^{2}$ is on the right. Its vertices correspond to the postcritical points of $f$. The vertex corresponding to $\infty$ is at infinity. The vertices of the triangle on the right correspond to $0, c_{R}$ and $c_{R}^{2}+c_{R}$ in counterclockwise order starting at the bottom. An edge on the left with label $n$ maps to the edge on the right with label $n$.

Step 1. $P_{2}=\left\{0, c_{R}, c_{R}^{2}+c_{R}, \infty\right\}$

Step 2. $P_{1}=\left\{ \pm c_{R}, \pm\left(c_{R}^{2}+c_{R}\right)\right\}$ 

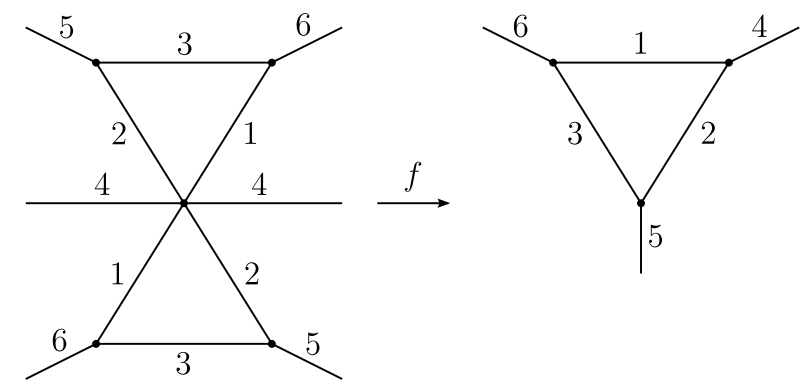

Figure 12. A finite subdivision rule for the rabbit $f$

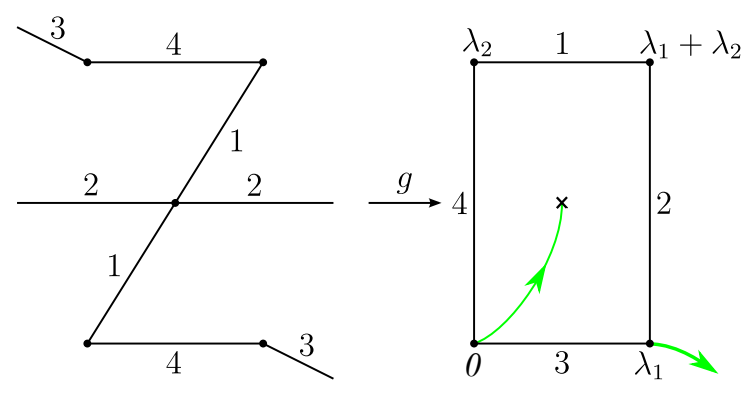

FiguRE 13. $\partial Q_{1}$ and its pullback under $g$

Step 3. We choose green arcs $\beta_{1}, \beta_{2}, \beta_{3}, \beta_{4}$ as in the right side of Figure 13. Two of these arcs are drawn as green arcs with arrows indicating the push direction. There are trivial arcs at the vertices labeled $\lambda_{2}$ and $\lambda_{1}+\lambda_{2}$.

Step 4. The quadrilateral $Q_{1}$ is drawn in the right side of Figure 13 .

Step 5. The complex $g^{-1}\left(\partial Q_{1}\right)$ is drawn in the left side of Figure 13. An edge with label $n$ on the left maps by $g$ to the edge with label $n$ on the right.

Step 6. To compute $\frac{p}{q}$, we view the edge on the right with label 1 as a core arc for $\gamma$. Pulling back this edge, we see that $\frac{p}{q}=1$ and $d=2$. To compute $\frac{r}{s}$, we view the edge on the right with label 4 as a core arc for $\delta$. Pulling back this edge, we see that $\frac{r}{s}=0$ and $e=1$.

Step 7. $A=\left[\begin{array}{cc}\frac{1}{2} & -1 \\ \frac{1}{2} & 0\end{array}\right]^{-1}=\left[\begin{array}{cc}0 & 2 \\ -1 & 1\end{array}\right]$

Step 8. $b=\lambda_{2}$

Step 9. We obtain the diagram in Figure 1.

Example 9.2. For a second example, we work through the algorithm to find a NET map presentation for

$$
f(z)=\frac{3 z^{2}}{2 z^{3}+1}
$$

This is the main example in Lodge's thesis [9]. It also appears in Section 4 of [1] and Example 3.2 of [3].

Note that $f(\omega z)=\omega^{2} f(z)$, where $\omega=e^{2 \pi i / 3}$. We compute:

$$
f^{\prime}(z)=\frac{6 z\left(2 z^{3}+1\right)-3 z^{2} 6 z^{2}}{\left(2 z^{3}+1\right)^{2}}=\frac{6 z\left(1-z^{3}\right)}{\left(2 z^{3}+1\right)^{2}} .
$$




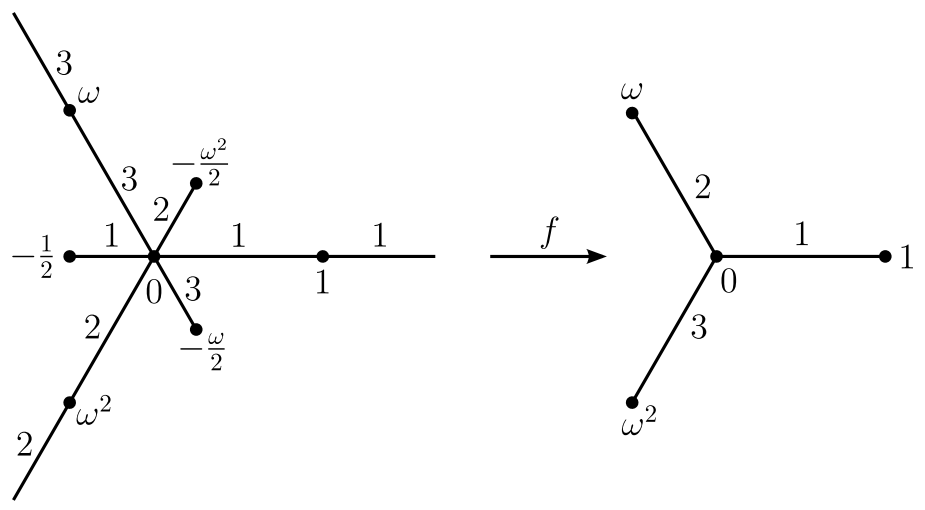

FiguRE 14. A finite subdivision rule for $f$

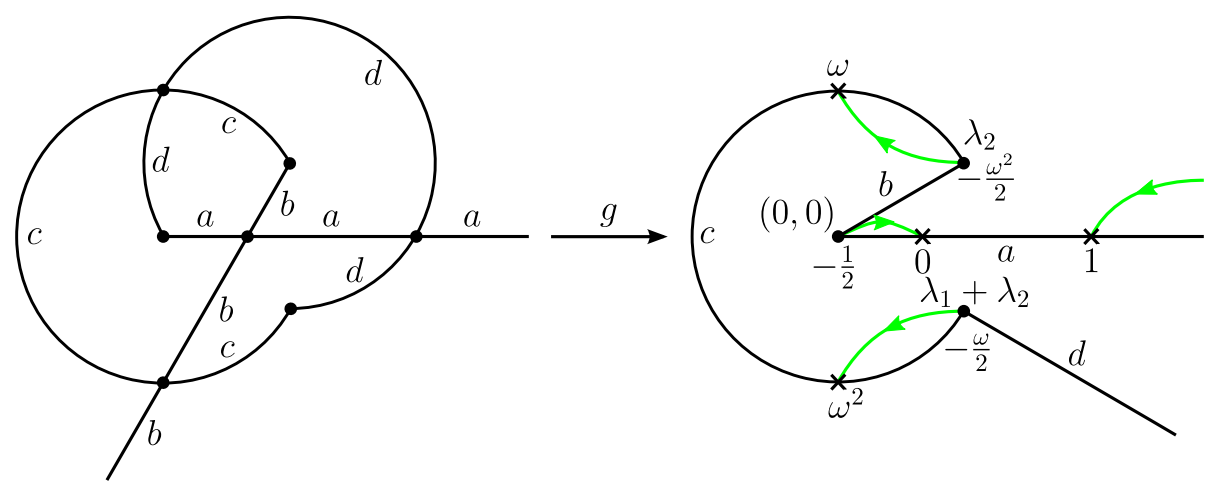

Figure 15. The boundary of the quadrilateral $Q_{1}$ and its pullback under $g$

The critical points of $f$ are $0,1, \omega$ and $\omega^{2}$. They are all simple. The postcritical points of $f$ are $0,1, \omega$ and $\omega^{2}$. So $f$ is indeed a NET map. We have the following table of values.

\begin{tabular}{c|cccccccc}
$z$ & $\infty$ & 0 & 1 & $\frac{-1}{2}$ & $\omega$ & $\frac{-\omega}{2}$ & $\omega^{2}$ & $\frac{-\omega^{2}}{2}$ \\
\hline$f(z)$ & 0 & 0 & 1 & 1 & $\omega^{2}$ & $\omega^{2}$ & $\omega$ & $\omega$
\end{tabular}

We see that $f$ is decreasing on the intervals $(-\infty, 0)$ and $(1, \infty)$ and that $f$ is increasing on the interval $(0,1)$. We conclude that $f$ maps $\left[-\frac{1}{2}, \infty\right]$ to $[0,1]$ in 3 -to- 1 fashion. This proves that the subdivision rule presentation of $f$ in Figure 14 is correct. The label of an edge on the left is the label of its image edge on the right.

We are prepared to apply the algorithm to find a NET map presentation for $f$.

Step 1. $P_{2}=\left\{0,1, \omega, \omega^{2}\right\}$

Step 2. $P_{1}=\left\{-\frac{1}{2},-\frac{\omega}{2},-\frac{\omega^{2}}{2}, \infty\right\}$

Step 3. We construct green $\operatorname{arcs} \beta_{1}, \beta_{2}, \beta_{3}, \beta_{4}$ in $S^{2}$ as in Figure 15. Arrows indicate push directions. In the right part of Figure 15, elements of $P_{1}$ are marked with dots and elements of $P_{2}$ are marked with $\times$ 's.

Step 4. We construct the quadrilateral $Q_{1}$ so that its boundary is the union of the black line segments and circular arc in the right side of Figure 15. We label the vertices of $Q_{1}$ as shown in Figure 15. The label $\lambda_{1}$ is at $\infty$. 


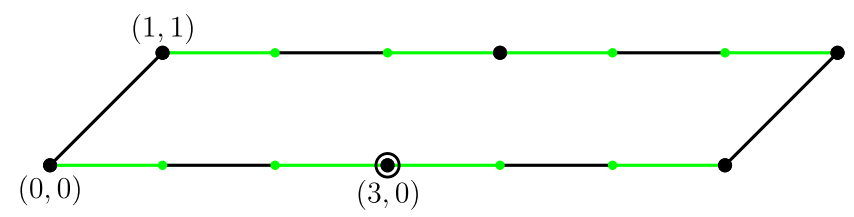

FiguRE 16. A presentation diagram for $f$

Step 5. The complex $g^{-1}\left(\partial Q_{1}\right)$ appears in the left side of Figure 15, correct up to isotopy rel $P_{1} \cup P_{2}$. Its edges are labeled with letters. The label of an edge is the label of its image in $\partial Q_{1}$.

Step 6. The interval $[-1 / 2, \infty]$ has label $a$ in the right side of Figure 15 . It is a core arc for a simple closed curve $\gamma$ in $S^{2} \backslash P_{1}$ with slope 0 . Figure 15 shows that $g^{-1}(\gamma)$ consists of one connected component with slope 0 . So $\frac{p}{q}=0$ and $d=3$. The line segment in the right side of Figure 15 with label $b$ is a core arc for a simple closed curve $\delta$ in $S^{2} \backslash P_{1}$ with slope $\infty$. Using Figure 15, we find that $g^{-1}(\delta)$ consists of one connected component with slope -3 . So $\frac{r}{s}=-3$ and $e=3$.

Step 7. To get a matrix with positive determinant, we take

$$
A=\left[\begin{array}{cc}
\frac{q}{d} & \frac{s}{e} \\
\frac{p}{d} & \frac{r}{e}
\end{array}\right]^{-1}=\left[\begin{array}{cc}
\frac{1}{3} & -\frac{1}{3} \\
0 & 1
\end{array}\right]^{-1}=\left[\begin{array}{ll}
3 & 1 \\
0 & 1
\end{array}\right] .
$$

Step 8. We see that $b=\lambda_{1}$.

Step 9. We obtain the NET map presentation diagram in Figure 16.

\section{REFERENCES}

[1] X. Buff, A. Epstein, S. Koch, and K. Pilgrim, On Thurston's pullback map, In Complex DynamicsFamilies and Friends, pp. 561-583. A. K. Peters, Wellesley, MA 2009.

[2] L. Bartholdi and V. Nekrashevych, Thurston equivalence of topological polynomials, Acta Math. 197 (2006), 1-51.

[3] J. W. Cannon, W. J. Floyd, W. R. Parry and K. M. Pilgrim, Nearly Euclidean Thurston maps, Conform. Geom. Dyn. 16 (2012), 209-255 (electronic).

[4] A. Douady and J. H. Hubbard, A proof of Thurston's topological characterization of rational functions, Acta Math. 171 (1993), 263-297.

[5] Benson Farb and Dan Margalit, A Primer on Mapping Class Groups, Princeton Univ. Press, Princeton, 2012.

[6] S. Koch, K. Pilgrim, and N. Selinger, Pullback invariants of Thurston maps, Trans. AMS 368(2016), no. 7, pp. 4621-4655.

[7] W. Floyd, G. Kelsey, S. Koch, R. Lodge, W. Parry, K. M. Pilgrim, E. Saenz, Origami, affine maps, and complex dynamics. Preprint, https://arxiv.org/abs/1612.06449.

[8] W. J. Floyd, W. R. Parry and K. M. Pilgrim, Modular groups, Hurwitz classes and dynamic portraits of NET maps, in preparation.

[9] R. Lodge, Boundary values of the Thurston pullback map, Ph.D. Thesis, Indiana University, 2012.

[10] V. Nekrashevych, Self-Similar Groups, Math. Surveys and Monographs 117, Amer. Math. Soc., Providence, 2005.

[11] The NET map web site, www.math.vt.edu/netmaps/. 
Department of Mathematics, Virginia Tech, Blacksburg, VA 24061, USA

E-mail address: floyd@math.vt.edu

URL: http://www.math.vt.edu/people/floyd

E-mail address: walter.parry@emich.edu

Department of Mathematics, Indiana University, Bloomington, IN 47405, USA

E-mail address: pilgrim@indiana.edu 